\title{
Structure of 50 to 70S RNA from Moloney Sarcoma Viruses
}

\author{
JAN MAISEL, $\dagger$ WELCOME BENDER, $\dagger$ SYLVIA HU, $\dagger \dagger \dagger$ P. H. DUESBERG, ${ }^{1}$ AND \\ NORMAN DAVIDSON ${ }^{2 *}$ \\ Department of Molecular Biology and Virus Laboratory, University of California at Berkeley, Berkeley, \\ California 94720,' and Department of Chemistry, California Institute of Technology, Pasadena, \\ California $91125^{2}$
}

Received for publication 26 July 1977

The 50 to $70 \mathrm{~S}$ RNAs of two clonal isolates of defective Moloney sarcomaleukemia helper virus complex were analyzed by gel electrophoresis and electron microscopy. The RNAs extracted from both clone 3 and clone 124-5R of Moloney sarcoma-leukemia virus complex contained some large monomer subunits ca. 10,000 nucleotides in length (10 kilobases), which are believed to be the Moloney leukemia virus subunits. Both RNAs had an excess of a smaller, sarcoma-specific subunit, 5 kilobases (clone 3) or 6 kilobases (clone 124-5R) in length. Electron microscopy of intact 50 to $70 \mathrm{~S}$ dimer RNA molecules showed for both clones many dimers of two small subunits, some dimers of two large subunits, but few if any heterodimers with one large and one small subunit. This result was unexpected because the sequences near the $5^{\prime}$ end of the RNA subunits, which are believed to be involved in the dimer linkage, are probably homologous between the large and small subunits. We also observed that some small-small dimers migrated anomalously slowly on nondenaturing gels. The nature of this slow-migrating complex is unknown; it could be a higher aggregate of the smallsmall dimer with additional small or large subunits, or it could be an extended conformation of the small-small dimer.

Cloned RNA tumor viruses contain a 60 to 70S RNA complex consisting of two very similar or identical 30 to $40 \mathrm{~S}$ subunits $(4,5,8,22)$. In several mammalian type C RNA tumor viruses, the two 30 to $40 \mathrm{~S}$ subunits are joined at their $5^{\prime}$ ends $(6,12,17,18)$. The function of the dimer molecules is not understood, but it has been proposed that viral heterozygotes, perhaps containing heterodimer RNA complexes consisting of two genetically distinct subunits, are obligate intermediates in recombination $(4,27,29)$. However, such heterodimer RNAs have thus far not been detected in virus released by cells infected with two or more different viruses.

Linkage of RNA subunits in a 50 to 70S RNA complex is thought to result from base pairing $(13,14,23)$ and possibly involves a short selfcomplementary sequence (18) near the $5^{\prime}$ end of the RNA (6). Accordingly, heterodimer formation might be limited to closely related virus strains whose RNAs share nucleotide sequences involved in dimer formation.

† Present address: Department of Pediatrics, University of California, San Francisco, CA 94143.

†† Present address: Department of Biochemistry, Stanford University, School of Medicine, Stanford, CA 94305.

tt† Present address: Department of Microbiology, University of Southern California, School of Medicine, Los Angeles, CA 90033.
In the Friend virus complex, which has several different RNA components $(12,20,26)$, it was observed that heterodimers were not common, but the length differences among the different RNA subunits are not much greater than the expected variation in length measurement for a homogeneous species, and the possibility of rare heterodimers could not be excluded.

In this study, the RNAs of two Moloney murine sarcoma-leukemia virus isolates (MSV) were examined for heterodimers. The system is advantageous for two reasons. First, the MSV virus complex contains two classes of RNA subunit of significantly different size, a 10-kilobase (kb) leukemia virus-specific RNA, termed the $\mathrm{L}$ subunit, and a 5- to 6-kb sarcoma virus-specific RNA, termed the $S$ subunit $(5,19,20)$. LS heterodimers should be readily distinguished from LL and SS homodimers, especially since dimer molecules containing broken subunits can be excluded visually in the electron microscope assay by labeling the $3^{\prime}$-polyadenylate [poly(A)] ends of intact RNAs (6). Secondly, the sarcoma virus-specific $\mathrm{S}$ subunit shares extensive sequence homology with the L subunit of Moloney leukemia virus (MLV; 7, 11, 24). Moreover, mapping of the homology between the $S$ subunits of a related strain (clone 124) of MSV and L subunits of MLV by electron microscopy of het- 
eroduplex molecules (17) and by biochemical methods (K. L. Beemon, D. Dina, and P. H. Duesberg, unpublished data) has indicated that the two molecules share sequences near their $5^{\prime}$ ends, where the dimer linkage appears to be located.

We report here that, when examined by electron microscopy, the 50 to 70S RNAs of clone 3 and clone 124-5R MSV are found to contain both SS and LL homodimers, but that there are no detectable LS heterodimers. However, gel electrophoresis of viral RNA under nondenaturing conditions shows that, whereas there are LL dimers and SS dimers with mobilities as expected for their respective lengths, there is also another kind of complex that has an electrophoretic mobility close to that of LL dimers. The structure of this third component is not known, but it includes SS dimers.

\section{MATERIALS AND METHODS}

Cells and viruses. MSV clone 3 (20) was isolated by infection of rat NRK cells with a tissue culturederived stock (HT-1) of MSV virions obtained from A. Hackett (9), followed by cloning in microtiter wells (20). A line of TB mouse cells infected with MSV clone 124 (3) was a gift of J. K. Ball; this virus was derived from an MSV stock provided by J. B. Moloney. Virus from these cells was plated at low multiplicity on rat NRK cells, a nonproducing focus was isolated and superinfected with MLV, and these cells produced virus (MSV clone 124-5R) containing RNA subunits of two sizes equivalent to those of MLV and MSV clone 124 (19). 3T3 (NIH) mouse cells infected by MLV were obtained from D. Smotkin. All cells were grown in Eagle minimal essential medium containing $10 \%$ fetal bovine serum, $0.5 \mu \mathrm{g}$ of Fungizone per ml, $100 \mathrm{U}$ of penicillin per $\mathrm{ml}$, and $50 \mu \mathrm{g}$ of streptomycin per ml.

The following observations show that the RNAs used were not substantially contaminated with other RNA components. A complementary DNA probe prepared from clone 1 MLV RNA protects $85 \%$ of clone 1 RNA and $85 \%$ and $58 \%$ of gel-purified L and S components of MSV clone 3, respectively, in hybridization and RNase digestion experiments (L. Evans and P. Duesberg, personal communication). The MLV RNA used has the expected complexity and fingerprint (5). The complexity of the S component of MSV clone 3 was determined by fingerprint analysis and gave the value expected for its molecular length $(J$. Maisel, personal communication). The S component of MSV clone 124-5R shows the same heteroduplex pattern with MLV clone 1 complementary DNA as was seen for MSV clone 124 RNA (17), showing that these RNAs are basically identical (Y. H. Chien, personal communication).

Gel electrophoresis. Polyacrylamide gels (2.1 or $1.9 \%)$ were prepared and analyzed as previously described (19).

Agarose gels were prepared in borate (2) or Trisacetate buffer (20). For denaturing gels, $5 \mathrm{mM}$ meth- ylmercury hydroxide was added to the borate gel solution (2).

RNA was eluted from polyacrylamide gels as previously described (21), and was recovered from agarose gels by the freeze-squeeze method (25). The RNA recovery by freeze-squeeze was lower than by elution (30\% versus ca. $80 \%$ ), but the RNA could be obtained quickly in a small volume, and it was essentially free of agarose. In some experiments, oligodeoxythymidylate-cellulose column fractionation (28) was used to remove from electrophoretically purified RNA preparations any RNA species that had been nicked near the $3^{\prime}$ end and therefore no longer contained poly(A).

Electron microscopy. RNA samples were treated with glyoxal, dialyzed, incubated briefly with simian virus 40 (SV40)-polydeoxythymidylate [poly (dT)] or SV40-polybromodeoxyuridine [poly(BUdR)], and spread by the formamide cytochrome $c$ method as described (6). [We now use SV40-poly(BUdR) instead of SV40-poly(dT) as a poly (A) label, because of the greater stability of poly(BUdR) $\cdot \operatorname{poly}(\mathrm{A})$ duplexes.] The standard glyoxal treatment (1 M glyoxal-0.01 M potassium phosphate $\left[\mathrm{pH} \mathrm{7.0]}\right.$, at $37^{\circ} \mathrm{C}$ for $1 \mathrm{~h}$ ) dissociated most of the dimers of these murine viruses. Therefore, less harsh glyoxal treatments (incubations for only $20 \mathrm{~min}$ or in the presence of $0.1 \mathrm{M} \mathrm{NaCl}$ ) were used to preserve the dimer linkage. The SV40 circles were used as an internal length standard, and the sizes of RNA molecules were calibrated by comparison with Escherichia coli 23S rRNA (1 $\mu \mathrm{m}=4.1$ kb).

\section{RESULTS}

Gel electrophoresis of clone 3 MSV RNA. RNA extracted from clone $3 \mathrm{MSV}$ virus contains two sizes of subunit. When 50 to $70 \mathrm{~S}$ viral RNA is heat denatured and analyzed by electrophoresis on a $2 \%$ polyacrylamide gel, two well-separated peaks are seen (20). The larger species comigrates with heat-denatured MLV and is identified as the leukemia (L) subunit. The smaller component $(\mathrm{S})$ is presumed to be the monomer unit of the genome of the defective sarcoma virus. Molecular lengths of $10.1 \pm 0.5$ $\mathrm{kb}$ and $5.1 \pm 0.3 \mathrm{~kb}$ for the $\mathrm{L}$ and $\mathrm{S}$ subunits were estimated by agarose gel electrophoresis in the presence of the strong denaturing agent $\mathrm{CH}_{3} \mathrm{HgOH}$ (2) (Fig. 1).

MSV 50 to 70S RNA that has not been denatured gives a single broad peak when sedimented in a sucrose gradient $(9,20)$. However, the high-molecular-weight RNA could be resolved into two components, I and II, when run on a nondenaturing agarose or acrylamide gel (Fig. 2A). MLV 50 to 70S RNA had an electrophoretic mobility on these gels that was nearly identical to that of the slower-migrating MSV component I. RNA from the two peaks of the nondenaturing gels was extracted, heat denatured, and applied to $2 \%$ acrylamide gels. The faster-migrating peak II gave after denaturation only S monomers (Fig. 2B), but the slower peak 


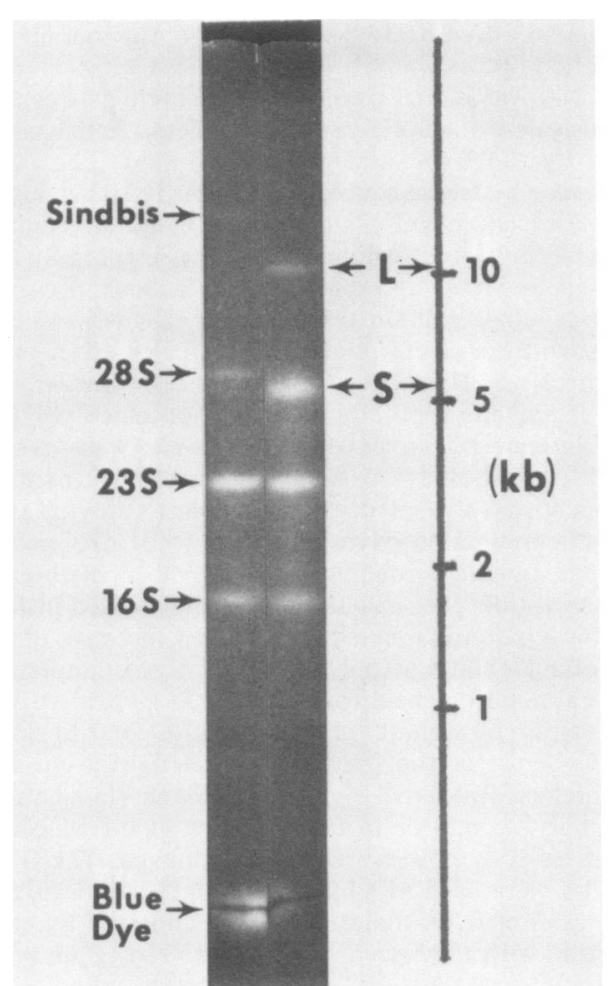

FIG. 1. Molecular weight determination of viral RNAs by electrophoresis in 1\% agarose gels in the presence of $6 \mathrm{mM} \mathrm{CH} \mathrm{CH}_{3} \mathrm{HOH}$. The left gel contained Sindbis viral $\left[{ }^{3} \mathrm{H}\right] \mathrm{RNA}, \mathrm{HeLa} 28 \mathrm{~S}$ ribosomal RNA, and $E$. coli $23 S$ and $16 S$ ribosomal RNAs (molecular lengths, $13.5,5.5,3.1$, and $1.7 \mathrm{~kb}$, respectively). The right gel had the same markers plus ${ }^{32} P$-labeled clone 3 MSV RNA. After being stained with ethidium bromide and photographed, the gels were sliced in 2mm sections and counted.

I contained subunits of both the $\mathrm{L}$ and $\mathrm{S}$ sizes (Fig. 2C).

The results on the composition of the two peaks were confirmed by cutting peak I and peak II slices from a nondenaturing agarose gel, polymerizing them directly onto a denaturing $\mathrm{CH}_{3} \mathrm{HgOH}$ gel, and subjecting them to electrophoresis in the second gel. By this technique, over $85 \%$ of the material in the slices from the first gel was recovered in the second gel, thus showing that the results in Fig. 2B and 2C were not distorted by possible inefficient recovery from the nondenaturing gel of Fig. 2A.

Electron microscopy of clone 3 MSV

RNA. The structures of the several components present in clone 3 MSV RNA were studied by electron microscopy. Because peak I had both $L$ and $S$ subunits, we were particularly interested in the question of whether any LS heterodimers existed.
Viral RNA was glyoxal treated and spread from formamide, as previously described (18). SV40 double-stranded circular DNA with poly $(\mathrm{dT})$ tails was hybridized to the viral RNA before spreading, to label the $3^{\prime}$-poly(A) ends (6). The dimers of MSV were mostly dissociated by the standard glyoxal treatment $(1.0 \mathrm{M}$ glyoxal-0.01 $\mathrm{M}$ potassium phosphate [ $\mathrm{pH} 7.0]$,

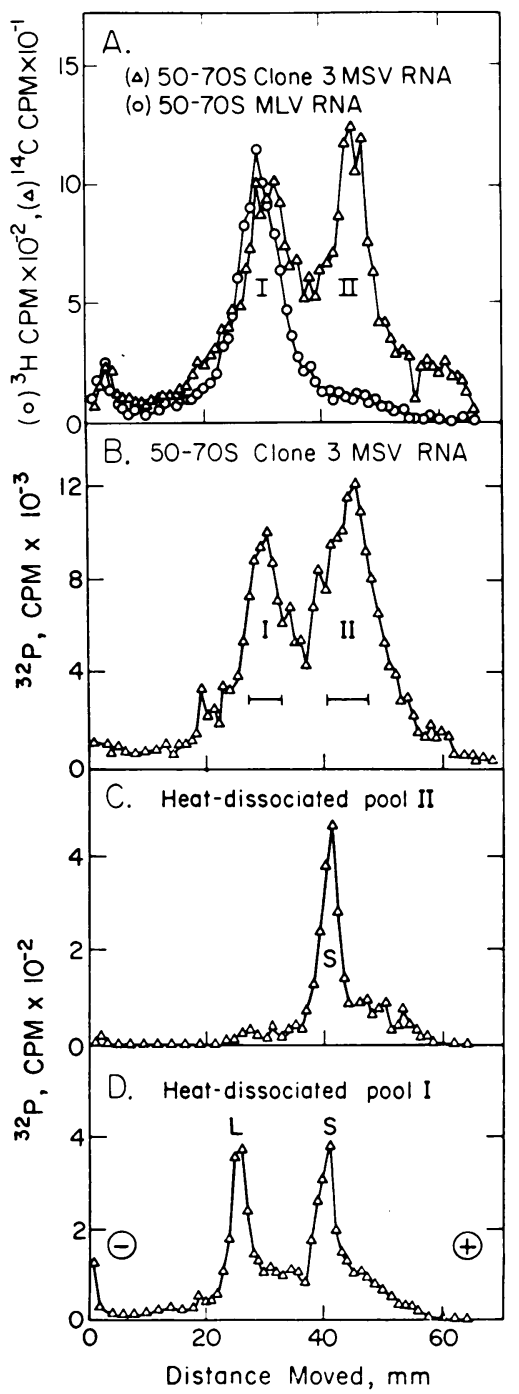

Fig. 2. Gel electrophoresis of the two components of 50 to TOS RNA of clone $3 \mathrm{MSV}$. (A) Clone 3 [2P]RNA and MLV [ $\left.{ }^{3} \mathrm{H}\right] \mathrm{RNA}$ were subjected together to electrophoresis in a $1.9 \%$ polyacrylamide gel. (B) Clone 3 [ ${ }^{2}$ P]RNA was subjected to electrophoresis as in (A), but the gel slices were counted by Cerenkov radiation. The slices indicated by the horizontal bars were pooled and eluted, and the re. covered RNA was heat denatured and rerun on $2.1 \%$ polyacrylamide gels $(C, D)$. 
at $37^{\circ} \mathrm{C}$ for $1 \mathrm{~h}$ ); therefore, $0.1 \mathrm{M} \mathrm{NaCl}$ was added to the solution to stabilize the dimer linkage structure during glyoxal treatment.

As already reported (17), the principal structure found in 50 to 70S RNA from MLV alone is the LL dimer. This dimer has the same general structural features previously observed for other mammalian viral RNAs $(12,18)$.

In contrast, the 50 to $70 \mathrm{~S}$ RNA from MSV yielded two kinds of structures. The less abundant component had exactly the same secondary structure features as described previously for MLV RNA; such an LL dimer hybridized to SV40 poly (dT) is shown in Fig. 3A. The more abundant molecule, which must represent the defective-sarcoma component, is shown in Fig. 3B. Like the MLV RNA dimers, the SS dimer molecules displayed twofold symmetry with a central dimer linkage structure. The SS dimers were only about half as long as LL dimers. The secondary structure loops in the centers of the $\mathrm{S}$ monomer units were also quite different. These loops were small figure-eight structures, often with a short hairpin near the midpoint of the loop. Schematic representations of the structures of both components and the contour lengths of the several features are given in Fig. 4.

In the preparations from MSV 50 to 70S RNA, we saw only LL and SS dimers and no LS heterodimers. To study this question critically, we photographed and measured about 50 randomly selected dimers that had clear dimer linkage structures and that had two unbroken subunits both ending in a poly(A) sequence hybridized to an SV40-poly(dT) label. Figure 5 is a plot of the length of the shorter subunit of each dimer versus the length of the longer subunit. The analysis of this kind of a plot has been discussed previously (12). A heterodimer molecule would be represented by a point that deviated significantly from the $45^{\circ}$ line in the plot. The vast majority of molecules in Fig. 5 are LL or SS dimers with subunits of almost equal length. There are a few molecules with one unusually short subunit; these presumably are broken subunits whose ends accidentally lie close to an SV40 circle, but there are none with the dimensions expected of a heterodimer.

In subsequent studies, we observed over 300 clone $3 \mathrm{MSV}$ dimers; of these, 3 molecules had the structure expected for an LS heterodimer, but only 1 (Fig. 3C) was attached at both outside ends to SV40-poly(dT), thus showing that it was unbroken. No certain conclusions can be drawn from a single rare molecule; for example, the molecule could possibly have been a broken or partially collapsed LL dimer. However, this molecule did appear to be a true heterodimer both by the contour lengths of the two subunits and by the positions and shapes of the two loops.

It seemed possible that heterodimers might exist but have a much lower stability than homodimers and thus be disrupted by our spreading conditions and not be scored. We therefore separated 50 to 70S MSV RNA on a nondenaturing agarose gel as in Fig. 2A, isolated RNA from each peak, treated it with glyoxal in high salt, and then mounted it for microscopy. Samples from the fast-migrating peak II gave SS dimer and S monomers, with occasional broken MLV fragments observed. Extensive searching of the sample from the slow-migrating peak I yielded four types of molecules: LL dimer, L monomer, SS dimer and S monomer. The ratios obtained from two independent experiments are listed in Table 1. These data confirm that there are significant amounts of SS and S components migrating anomalously in the slow peak. If unstable LS heterodimers do exist, the number of $\mathrm{L}$ and $\mathrm{S}$ monomers seen here requires that these heterodimers be a minor component. Finally, we wish to note that there is a certain number of tangled uninterpretable molecules on grids prepared by the gentle glyoxal treatments used here, so that the numbers in Table 1 may not be strictly representative.

Gel electrophoresis of clone $124 \mathrm{MSV}$. The RNA of clone 124, an MSV variant, was investigated in an attempt to resolve the question of whether the S subunits of MSV can form, in addition to homodimers, larger complexes consisting of more than two $S$ subunits, or whether both $S$ and L subunits are necessary to form such large complexes. The RNA of MSV clone 124, propagated in a mouse cell line, differs from that of MSV clone 3 in that it contains a large excess of $S$ over $L$ subunits, at an approximate mass ratio of 30:1 (19). Hence, if MSV clone $124 \mathrm{~S}$ subunits were able to form multimeric complexes as well as homodimers, the $\mathbf{5 0}$ to 70S RNA would consist of two electrophoretic species. If both $L$ and $S$ subunits were necessary to form complexes larger than S subunit homodimers, the 50 to 70S RNA of MSV clone 124 would consist of only one major 50 to 70S RNA species, namely, SS homodimers, because insufficient $L$ subunits are present in this virus stock to permit formation of larger complexes. The 50 to 70S RNA of clone 124 MSV consists essentially of only one major species, which migrates faster than 60 to 70S MLV RNA and is similar to peak II of MSV clone 3 RNA (Fig. 6A). After heat dissociation, the RNA was converted predominantly into a species containing single $S$ subunits that migrated faster than the $L$ subunits but a little slower than the $S$ subunits of MSV clone 3. This electrophoretic difference 

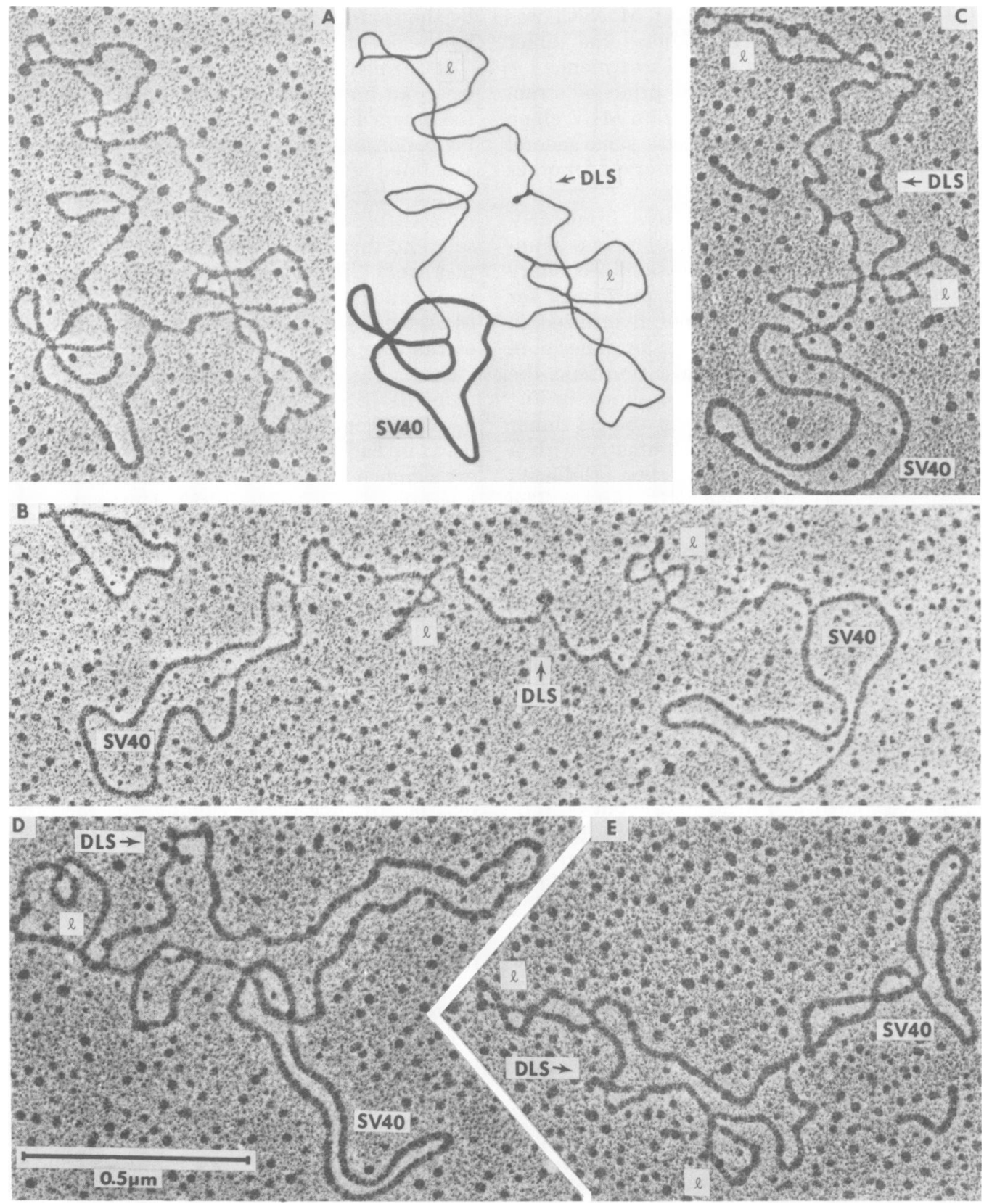

Fig. 3. Electron micrographs of MSV RNA dimers. (A), (B), and (C) show clone 3 RNA, glyoxal treated and spread from $40 \%$ formamide; in each case, both poly $(A)$ ends were hybridized to short poly $(d T)$ tails on an SV40 DNA circle. (A) Dimer of large subunits (LL); an interpretive tracing is shown. (B) Dimer of small subunits (SS). (C) Single example of an apparent heterodimer (LS); the loop at the upper left is similar in size and position to those of MLV subunits, and the lower right loop looks like a partially collapsed figureeight loop of an $S$ subunit. $(D, E)$ Clone 124-5R RNA dimers, glyoxal treated, spread from 50\% formamide, and attached to SV40 circles with poly(BUdR) tails. (D) LL dimer. (E) SS dimer. The SV40-poly(BUdR) preparation used here had few tails per circle; in both cases shown, both viral poly(A) tails have hybridized to a single poly $(B U d R)$ tail. DLS, The dimer linkage; l, the characteristic large loops. All pictures are at the same magnification. 

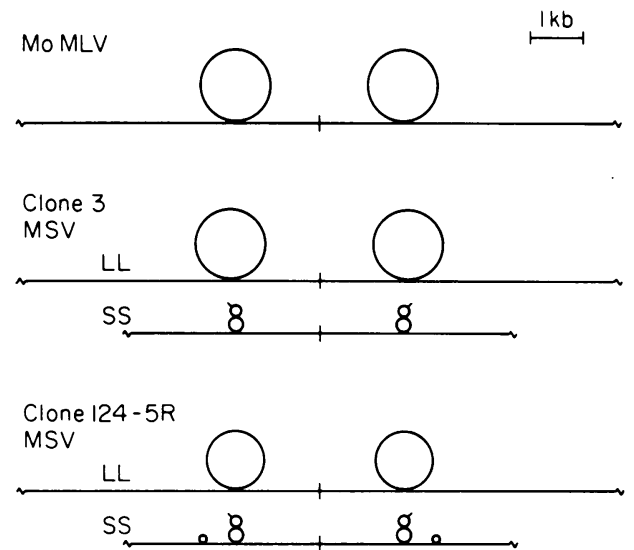

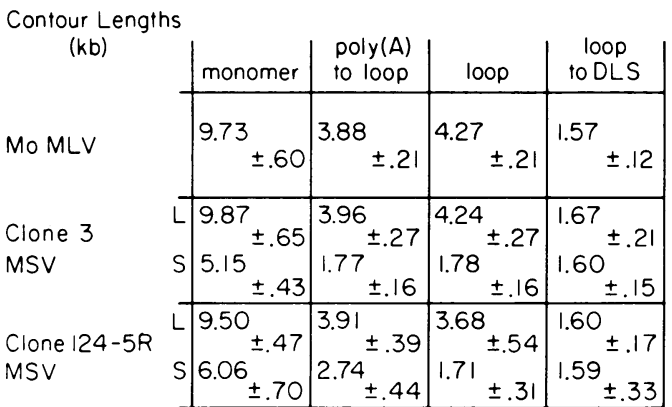

Fig. 4. Diagram of the structures of $M L V$ and $M S V$ dimers. The dimers of MLV and MSV clone 3 and clone 124-5R are all drawn to scale. The table lists contour lengths of the various subunits and their secondary structure features. The zig-zag lines at the ends of the molecules symbolize poly(A). The $S$ subunits of clone 124-5R sometimes show a small loop or hairpin between the figure-eight loop and the poly(A) end.

between the $S$ subunits of the two strains of MSV has been described previously (19; Fig. 4).

Clone 124-5R MSV RNA. A possible interpretation of the above result is that the SS dimers of MSV clone 124 do not form any slowly migrating complexes because $L$ subunits are necessary for this phenomenon and are present in insufficient amount. We have therefore studied the RNA from a cell line, 124-5R, derived from 124 ; it releases a larger amount of the $L$ component than does the parental cell line.

On a nondenaturing gel, the 50 to $70 \mathrm{~S}$ RNA of this virus complex was resolved into two peaks, I and II (Fig. 7A). After heat dissociation, 50 to 70 S clone 124-5R MSV RNA was resolved into two subunit species. In accord with a previous study (19), the larger L subunit comigrated with the L subunit of clone $3 \mathrm{MSV}$ and the smaller S subunit migrated more slowly than the $\mathrm{S}$ subunit of MSV clone 3 (Fig. 7B). The
RNA was also sized on $1 \%$ agarose gels in the presence of $5 \mathrm{mM} \mathrm{CH}_{3} \mathrm{HgOH}$; the $\mathrm{L}$ subunit ran as $9.7 \pm 0.5 \mathrm{~kb}$ and the $\mathrm{S}$ subunit was $6.1 \pm 0.3$ kb (data not shown). As with clone $3 \mathrm{MSV}$, gel electrophoretic analysis of heat-dissociated peak I RNA reveals both $\mathrm{L}$ and $\mathrm{S}$ subunits (Fig. 7C). The signal-to-noise ratio for these experiments is not as good as for the experiments with clone 3 MSV RNA in Fig. 2 and 3, because the ratio of peak I to peak II RNA is less and the separation between the two peaks is not as great;

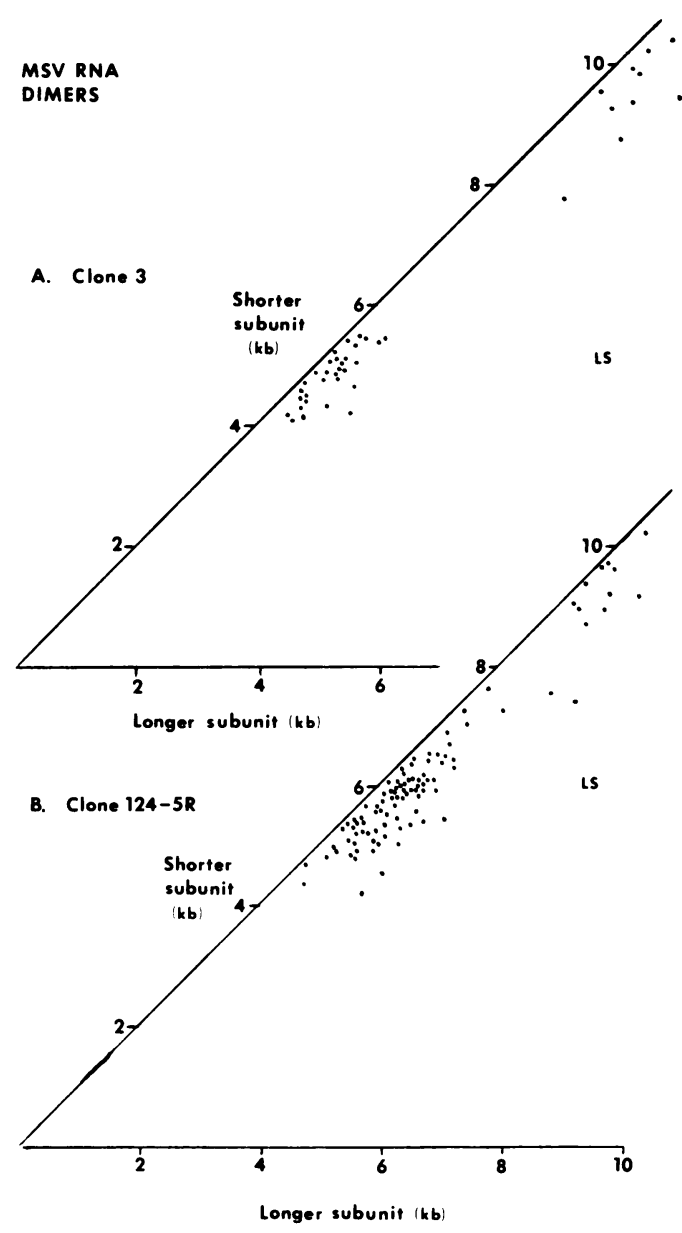

Fig. 5. Correlation of the two monomer lengths of MSV dimers. Randomly selected dimer molecules, each with a clear dimer linkage structure and with both poly(A) ends labeled, were photographed and measured. Each dot represents one dimer molecule; the horizontal coordinate is the length of its longer subunit, and the vertical coordinate is the length of its shorter subunit. $L L$ and SS homodimers lie near the diagonal, since both subunits are about the same length. LS heterodimers, had they been found, would be plotted around the position marked LS. (A) 41 clone 3 MSV dimers; (B) 98 clone 124-5R dimers. 
TABLE 1. Analysis of peak I RNA by electron microscopy ${ }^{a}$

\begin{tabular}{ccrccc}
\hline \multirow{2}{*}{ Expt } & \multicolumn{5}{c}{ No. of molecules } \\
\cline { 2 - 6 } & LL $^{b}$ & \multicolumn{1}{c}{$\mathrm{L}$} & $\mathrm{SS}$ & $\mathrm{S}$ & $\mathrm{L} / \mathrm{S}^{\mathrm{c}}$ \\
\hline 1 & 114 & 7 & 46 & 14 & 2.24 \\
2 & 145 & 231 & 65 & 60 & 2.74 \\
\hline
\end{tabular}

${ }^{a}$ Clone $3 \mathrm{MSV}$ was subjected to electrophoresis in a 1\% agarose gel, and the peak I gel slices were eluted by the freeze-squeeze method. The recovered RNA was glyoxal treated ( $1 \mathrm{M}$ glyoxal $-0.1 \mathrm{M} \mathrm{NaCl}$ at $37^{\circ} \mathrm{C}$, for $40 \mathrm{~min}$ [experiment 1] or $60 \mathrm{~min}$ [experiment 2]) and spread for electron microscopy; the grids were then scanned for LL, SS, and LS dimers and for $\mathrm{L}$ and $\mathbf{S}$ monomers. Two separate experiments were performed. Only three presumptive LS dimers were found in the whole search; the numbers of molecules in the other four classes are listed for the separate experiments. In experiment 2 , the longer glyoxal treatment disrupted many of the dimers.

${ }^{b}$ Molecule type.

${ }^{c}$ Ratio of total numbers of $\mathrm{L}$ and $\mathrm{S}$ units.

nevertheless, the results support the view that there are $\mathrm{S}$ as well as $\mathrm{L}$ subunits in peak $\mathrm{I}$.

For electron microscope studies, 50 to $70 \mathrm{~S}$ RNA from clone 124-5R MSV was glyoxal treated (in this case in $1 \mathrm{M}$ glyoxal-0.01 $\mathrm{M} \mathrm{PO}_{4}$ [pH 7.0] for $20 \mathrm{~min}$ ), hybridized with SV40poly(BUdR), and spread from $50 \%$ formamide. As with the clone 3 RNA, there were dimers with two large (9.5-kb) subunits and dimers with two smaller (6-kb) subunits. These dimers are diagramed in Fig. 4. The LL dimers of clone 124-5R were indistinguishable from those of MLV or clone $3 \mathrm{MSV}$ (Fig. 3D). The SS dimers (Fig. 3E) were identical in size and secondary structure to those of clone $3 \mathrm{MSV}$, except that the distance from the poly(A) end to the figureeight loop is $2.7 \mathrm{~kb}$ in clone 124-5R instead of $1.8 \mathrm{~kb}$ as in clone 3 . In the region between the poly(A) and the loop on clone 124-5R S subunits, there is often a small loop or hairpin about 0.6 $\mathrm{kb}$ in length and about $1.5 \mathrm{~kb}$ from the poly(A) end; it is conceivable that the clone 124-5R and clone $3 \mathrm{~S}$ subunits are identical except for an insertion or deletion of this $0.6-\mathrm{kb}$ sequence.

As with clone $3 \mathrm{MSV}$, we did not find LS heterodimers in clone 124-5R. About 100 randomly selected dimers were photographed and measured, taking only molecules with a clear dimer linkage and both ends attached to SV40poly(BUdR). The relative lengths of the two monomer units of each dimer pair are plotted in Fig. 5B. There are no molecules with the dimensions expected for heterodimers.

Further experiments. It was not clear whether the SS dimers found in peak I of clone $3 \mathrm{MSV}$ were there because of variation in conformation, because of nonspecific aggregation, or because of specific large complexes such as LSS trimers or $\mathrm{S}_{4}$ tetramers. Several experiments were done to test these possibilities.

It seemed possible that $\mathbf{S}$ subunits artificially aggregated with MLV RNA after the RNAs had been extracted from the respective virions. To test this possibility, ${ }^{32} \mathrm{P}$-labeled MSV clone 124 culture fluid was mixed with $\left[{ }^{3} \mathrm{H}\right]$ uridine-labeled MLV culture fluid, and their RNAs were extracted together. The 50 to $70 \mathrm{~S}$ MLV $\left[{ }^{3} \mathrm{H}\right] \mathrm{RNA}$ formed a distinct, more slowly migrating peak (Fig. 6B). The small amount of $\left[{ }^{32} \mathrm{P}\right] \mathrm{RNA}$ that migrated with the slower MLV $\left[{ }^{3} \mathrm{H}\right] \mathrm{RNA}$ was also present in clone 124 RNA extracted in the absence of MLV (Fig. 6A) and is thought to be helper MLV RNA present as a minor component $(11,19)$. We conclude that simultaneous extraction of MLV and MSV clone 124 RNA did not generate the type of hybrid RNA complex seen in clone 3 or clone 124-5R MSV. Therefore, the

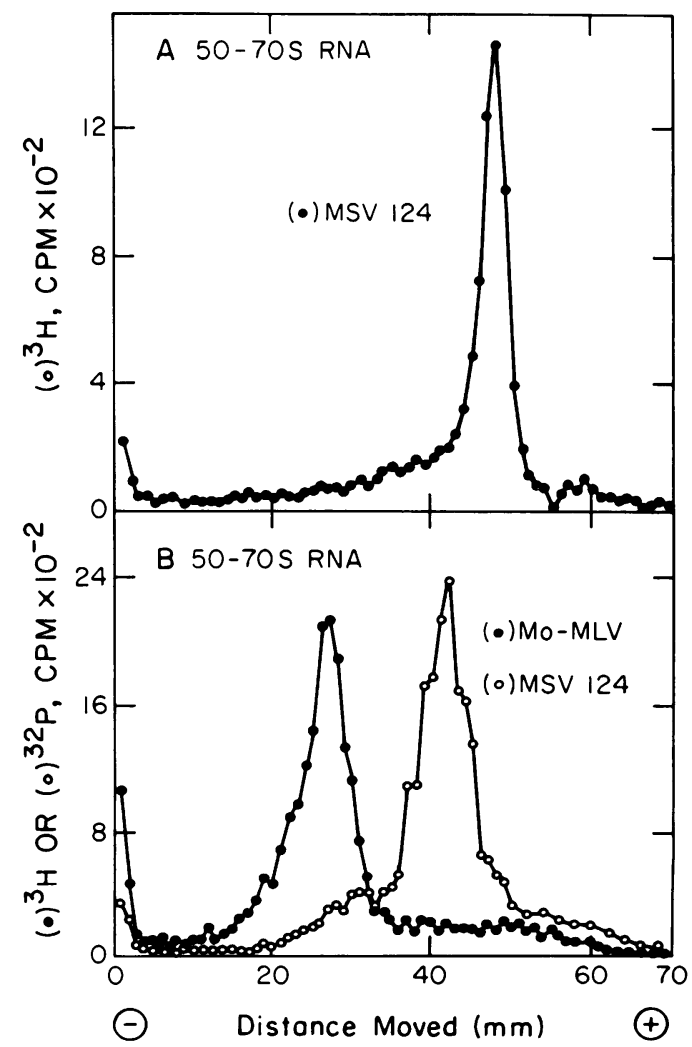

Fig. 6. Electrophoresis of clone $124 \mathrm{MSV}$ RNA. (A) Clone 12450 to $70 S$ RNA run on a $1.9 \%$ polyacrylamide gel. (B) ${ }^{3} \mathrm{H}$-labeled $M L V$ (intact virus) was mixed with ${ }^{32} P$-labeled clone $124 M S V$ (intact virus); the mixture was phenol extracted and sedimented to prepare 50 to $70 S R N A$, and the mixed RNA was subjected to electrophoresis on a $1.9 \%$ polyacrylamide gel. 


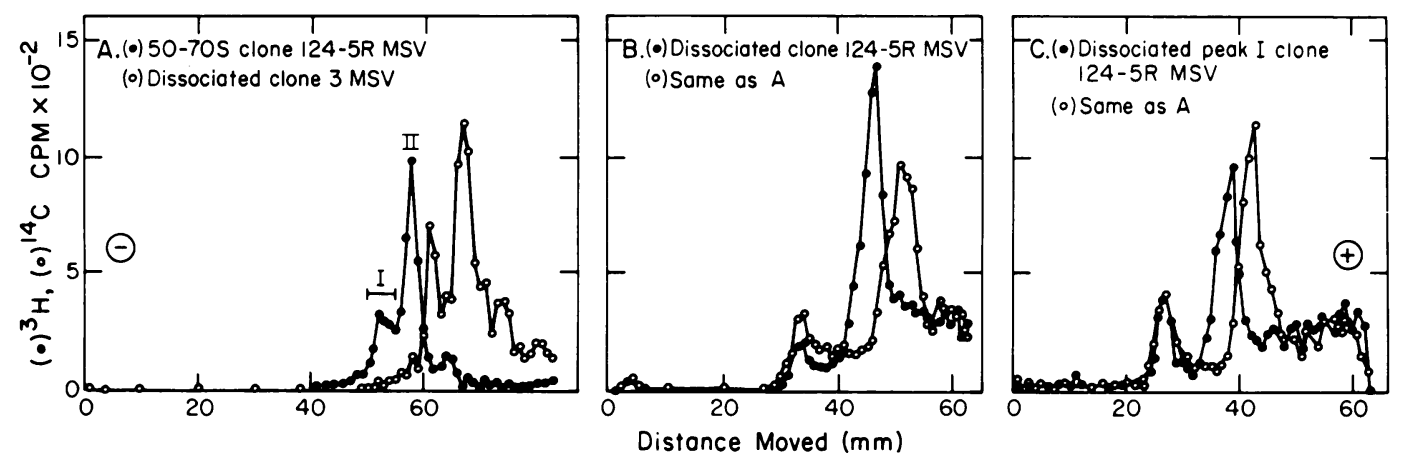

Fig. 7. Electrophoresis of clone 124-5R MSV RNA. (A) 50 to $\left.70 S{ }^{3} H\right] R N A$ of clone 124-5R MSV was mixed with heat-dissociated $\left[{ }^{14} \mathrm{C}\right] R N A$ of clone $3 \mathrm{MSV}$ and subjected to electrophoresis in a $1.6 \%$ agarose gel in Tris-acetate buffer. (B) 50 to $70 S\left[{ }^{3} \mathrm{H}\right] \mathrm{RNA}$ of clone 124-5R was mixed with 50 to $70 \mathrm{~S}\left[{ }^{14} \mathrm{C}\right] \mathrm{RNA}$ of clone $3 \mathrm{MSV}$; the mixture was heat dissociated and subjected to electrophoresis in a $2 \%$ polyacrylamide gel. (C) Each 1-mm gel slice of (A) was melted $\left(1 \mathrm{~min}\right.$ at $\left.100^{\circ} \mathrm{C}\right)$ in $100 \mu \mathrm{l}$ of $0.2 \times$ Tris-acetate buffer, and its

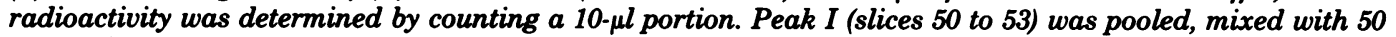
to $70 \mathrm{~S}\left[{ }^{4} \mathrm{C}\right] \mathrm{RNA}$ of clone $3 \mathrm{MSV}$, heated at $100^{\circ} \mathrm{C}$ for $45 \mathrm{~s}$, and rerun on a $2 \%$ polyacrylamide gel.

presence of $S$ subunits in peak $I$ in clone 3 and clone 124-5R RNAs is not an artifact of the RNA extraction and handling.

In an attempt to resolve possibly heterogeneous RNA complexes present in peak I, we subjected clone 3 MSV RNA to electrophoresis in agarose gels under partially denaturing conditions. It has been shown that 60 to $70 \mathrm{~S}$ Rous sarcoma virus RNA is dissociated into 30 to $40 \mathrm{~S}$ subunits at room temperature by $8 \mathrm{M}$ urea at low ionic strength (10). Clone 350 to 70S RNA was not dissociated into subunits when subjected to electrophoresis in agarose gels containing up to $6 \mathrm{M}$ urea. In the presence of $6 \mathrm{M}$ urea, peak I appeared slightly broader than in the absence of urea, but RNA eluted from both sides of the $6 \mathrm{M}$ urea peak I still contained S subunits (data not shown).

The stabilities of the several components of clone 3 MSV RNA were also tested by thermal melting. RNA was extracted from peaks I and II of a nondenaturing agarose gel, samples were dialyzed against $0.1 \mathrm{M} \mathrm{NaCl}-0.01 \mathrm{M}$ Tris- 0.001 $M$ EDTA (pH 8.5), the dialyzed samples were heated to various temperatures for $2 \mathrm{~min}$, and the samples were rerun on nondenaturing agarose gels. MLV dimers and the SS dimers of peak II both melted into the respective monomer units in the temperature interval from 55 to $65^{\circ} \mathrm{C}$. Peak I melted into subunits in the same temperature range, and there was no conversion of peak I material to any faster-migrating components by heating at $50^{\circ} \mathrm{C}$ (data not shown).

A secondary structure feature in $L$ monomers. In the course of the electron microscopic examination, we noted an unusual secondary structure feature of the L subunit RNA produced by heating and quenching. Clone $3 \mathrm{MSV}$
50 to 70 S RNA was dissociated by heating (58\% formamide- $0.006 \mathrm{M}$ EDTA-0.06 $\mathrm{M}$ Tris [ $\mathrm{pH}$ 8.5] at $40^{\circ} \mathrm{C}$ for $1 \mathrm{~min}$ ) and quenched on ice. Samples were mounted from $55 \%$ formamide solution onto an $18 \%$ formamide hypophase. After this treatment, the RNA was all of monomer length, and the subunits all lacked the usual loops. The $\mathrm{S}$ monomers appeared as simple linear molecules. However, the $\mathrm{L}$ monomers displayed a new loop and stem structure of contour length $1.3 \pm 0.1 \mathrm{~kb}$ beginning $0.9 \pm 0.1 \mathrm{~kb}$ from the $5^{\prime}$ end (Fig. 8). The large loops typical of the $\mathrm{L}$ subunits in the dimer never reformed. In rare molecules, the dimer linkage was intact, with two of the new hairpins on either side. This showed that the hairpin feature is close to the $5^{\prime}$ end of the $L$ monomer. The large loop in the native molecule begins at $1.6 \mathrm{~kb}$ from the $5^{\prime}$ end. The sequence to which it is evidently paired is at $5.9 \mathrm{~kb}$. The RNA sequences of the secondary structure feature seen in heated and quenched RNA map at 0.9 to $2.2 \mathrm{~kb}$ from the $5^{\prime}$ end. Thus, the loop structure and the hairpin in the monomer may be alternative base-paired structures for this particular region. This structural transition also occurs when the 50 to $70 \mathrm{~S}$ RNAs of other mammalian RNA tumor viruses, such as RD-114 and baboon endogenous virus, are dissociated and rapidly quenched (19).

\section{DISCUSSION}

The experiments described here indicate that the 50 to 70S RNAs of MSV clones 3 and 1245R consist of two classes of RNA complex, i.e., SS and LL homodimers. LS heterodimers were absent or comprised less than $1 \%$ of the dimer complexes, as assayed by direct visualization in the electron microscope, whereas, on the basis 


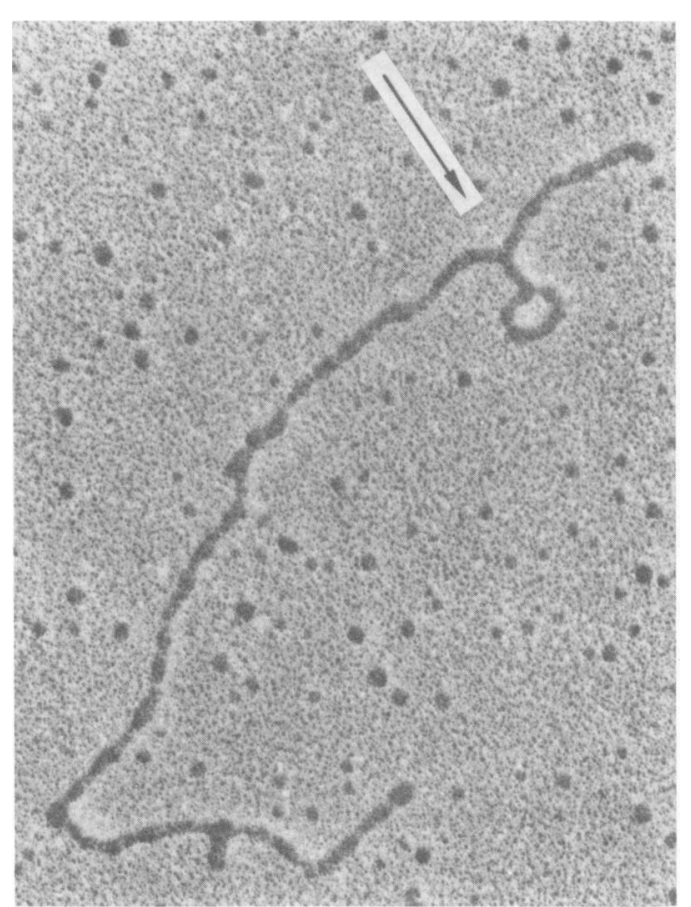

Fig. 8. A secondary structure feature in $L$ monomers produced by heating and quenching. Clone 3 MSV 50 to $70 S$ RNA was dissociated by heating in $58 \%$ formamide-60 mM Tris-6 mM EDTA (pH 8.5) at $40^{\circ}$ for $1 \mathrm{~min}$, quenched on ice, and spread from 55\% formamide. A new secondary structure feature $(\rightarrow)$ is observed on the $L$ subunits.

of random assortment, one would expect for the sample of molecules in Fig. 5A (ratio of $S$ to $L$ subunits of 3:1) that the relative fractions of SS, $\mathrm{LS}$, and LL dimers would be $0.56,0.38$, and 0.06 , respectively.

The signal-to-noise ratios in the various gel electrophoresis experiments were such that we cannot rigorously exclude the possibility that there is a modest fraction of LS heterodimers in the sample, but that these are dissociated much more readily than LL and SS dimers and are not seen under the partially denaturing conditions used for electron microscopy. However, gel electrophoresis experiments did not detect any complexes significantly less stable than LL and SS dimers. As discussed in more detail below, $\mathrm{L}$ and $\mathrm{S}$ monomer units probably have the same sequences at their $5^{\prime}$ ends, so that LL, SS, and LS dimers should all involve the same base pairs and therefore have the same stability.

Our other principal finding is contradictory to the simple view that the sample of 50 to $70 \mathrm{~S}$ MSV virion RNA consists only of SS and LL dimers. The gel electrophoresis studies showed that, under nondenaturing and mildly denatur- ing conditions, there are LL and SS dimers with mobilities as expected for their respective lengths; however, there is another kind of complex that has an electrophoretic mobility close to that of LL dimers but that contains SS dimers. No evidence for these special structures has been observed by electron microscopy.

The interaction responsible for the slow migration of some SS dimers is not an artifact of the extraction process. It appears to depend on synthesis of both $S$ and $L$ genomes in the same cell. The interaction does not melt out at a temperature substantially lower than the dissociation temperature of SS and LL homodimers.

Several hypotheses may be suggested to explain there results. (i) There is a conformational isomer of the SS dimers that is more extended and more slowly migrating in nondenaturing and even in $6 \mathrm{M}$ urea gels than the standard SS dimers themselves. (ii) There is a higher specific aggregate, perhaps an LSS or an $\mathrm{S}_{4}$ tetramer. These molecules form tangled structures that haven't been recognized and analyzed under the conditions used for electron microscopy. (iii) There is a general interaction resulting in higher aggregates involving, for example, SS and LL dimers. The interaction is slightly weaker than that responsible for dimerization, but the resolution of the urea gel and thermal melting experiments is insufficient to reveal this. (iv) $\mathrm{A}$ reviewer has suggested that higher aggregates of viral RNA may be formed when double-budded virions are released from cells (30).

We know of no decisive evidence to choose among these several possibilities, but the ratio of $\mathrm{L}$ monomers to SS dimers in experiment 1 of Table 1 argues against hypothesis (ii). We note that, whatever the nature of the slowly migrating S-containing species, its formation may be dependent on the cell type in which the virus grows and may be different, for example, in mouse TB cells (clone 124) than in rat NRK cells (clones 3 and 124-5R).

The findings that all known strains of MSV infect with double-hit kinetics $(1,15)$ argue against the existence of heterodimers, because virions containing an LS subunit in the same RNA complex might be expected to plate with single-hit kinetics. Also, no recombination between a mammalian sarcoma virus and its helper to give a nondefective sarcoma virus has been observed. This lack of recombination may be due to the absence of heterozygotes, if heterozygote formation is an obligatory step in RNA tumor virus recombination (P. K. Vogt, in Fraenkel-Conrat and R. R. Wagner [ed.], Comprehensive Virology, in press; 28,29$)$. Note, however, that, in the particular case of clone 124 MSV, the formation of a nondefective sarcoma 
virus would probably be impossible by homologous recombination. Near the $3^{\prime}$ end of clone $124 \mathrm{MSV}$, a new RNA sequence of $1.5 \mathrm{~kb}$ has been substituted in the RNA for a 2.9-kb region of the helper. Assuming that the new 1.5-kb sequence is essential for transformation and that the 2.9-kb region of the helper contains information essential for virus replication, each possible recombinant would have either one or the other sequence in the substitution region, and thus no nondefective sarcoma viruses would be formed (L.-H. Wang, P. H. Duesberg, T. Robins, H. Yakota, and P. K. Vogt, Virology, in press).

From the appearance of the dimer linkage structure of several mammalian viruses in cytochrome films, and from its appearance in gene 32 and other higher-resolution spreading methods (S. Hu and M. Wu, personal communication), we believe that the base-paired sequences involved in the dimer linkage structure probably lie somewhere within the first 600 nucleotides from the $5^{\prime}$ end. Heteroduplex analysis (17) indicates that clone 124 MSV RNA forms a duplex with MLV complementary DNA throughout the 2.25-kb region, starting at the $5^{\prime}$ end of the RNA. Analysis of RNase T1 oligonucleotides reveals no sequence differences in this region $(K$. L . Beemon, D. Dina, and P. H. Duesberg, private communication). Heteroduplexes between clone $3 \mathrm{MSV}$ and MLV have not yet been studied, but we expect to see the same homology regions, because both clone 3 and clone 124 are derivatives of J. B. Moloney's original MSV and because the two types of MSV subunit are so similar in secondary structure. Such a heteroduplex analysis cannot detect sequence mismatch below the level of about $10 \%$, nor can a short single-stranded region of RNA near the $5^{\prime}$ end of the MSV RNA be identified if the MLV complementary DNA is actually not quite full length. Therefore, it is probable, but not absolutely proven, that the $S$ and $L$ subunits have identical or very similar sequences involved in the dimer linkage.

If $L$ and $S$ subunits do have the same sequence at the $5^{\prime}$ end, and if dimer formation is simply due to base pairing in this region, as proposed by Kung et al. (18) or Haseltine et al. (16), one would expect to see LS heterodimers with the same stability as the homodimers.

As noted above, failure to form heterodimers may be due to small differences between $\mathrm{L}$ and $S$ subunits in the base-pairing sequences for dimerization. We think it is more likely that associations between two RNA subunits in regions other than $5^{\prime}$ ends are necessary for dimer formation and/or viral packaging. Alternatively, dimer formation may require that two neighboring RNA chains link during transcription.

\section{ACKNOWLFDGMINTS}

W. B. has been the recipient of a National Science Foundation fellowship and of a Public Health Service training grant from the National Institute of General Medical Sciences. This research has been supported by Public Health Service research grant CA-11426 from the National Cancer Institute and by Public Health Service contracts NO1-CP-61001 and NO1-CP-43306 with the Virus Cancer Program of the National Cancer Institute.

\section{LTERATURE CITED}

1. Aaronson, S. A., and W. P. Rowe. 1970. Non-producer clones of murine sarcoma virus-transformed BALB/373 cells. Virology 42:9-19.

2. Bailey, J. M., and N. Davidson. 1976. Methylmercury as a reversible denaturing agent for agarose gel electrophoresis. Anal. Biochem. 70:75-85.

3. Ball, J. K., J. A. McCarter, and S. M. Sunderland. 1973. Evidence for helper independent murine sarcoma virus. I. Segregation of replication-defective and transformation-defective viruses. Virology 56:268-284.

4. Beemon, K., P. Duesberg, and P. K. Vogt. 1974. Evidence for crossing over between avian tumor viruses based on analysis of viral RNAs. Proc. Natl. Acad. Sci. U.S.A. 71:4254-4258.

5. Beemon, K. L., A. J. Faras, A. T. Haase, P. H. Duesberg, and J. E. Maisel. 1976. Genomic complexities of murine leukemia and sarcoma, reticuloendotheliosis, and visna viruses. J. Virol. 17:525-537.

6. Bender, W., and N. Davidson. 1976. Mapping of poly(A) sequences in the electron microscope reveals unusual structure of type C oncornavirus RNA molecules. Cell 7:595-607.

7. Benveniste, R., and E. Scolnick. 1973. RNA in mammalian sarcoma virus transformed non-producer cells homologous to murine leukemia virus RNA. Virology 51:370-387.

8. Billeter, M. A., J. T. Parsons, and J. M. Coffin. 1974 The nucleotide sequence complexity of avian tumor virus RNA. Proc. Natl. Acad. Sci. U.S.A. 71:3560-3564

9. Bondurant, M. C., A. J. Hackett, and F. L. Schaffer. 1973. Infectivity and RNA patterns as functions of highand low-dilution passage of murine sarcoma-leukemia virus: evidence for autointerference within an oncornavirus population. J. Virol. 11:642-647.

10. Delius, H., P. H. Duesberg, and W. F. Mangel. 1975. Electron microscope measurements of Rous sarcoms virus RNA. Cold Spring Harbor Symp. Quant. Biol. 39:835-843.

11. Dina, D., K. Beemon, and P. H. Duesberg. 1976. The 30S Moloney sarcoma virus RNA contains leukemia virus nucleotide sequences. Cell 9:299-309.

12. Dube, S., H.-J. Kung, W. Bender, N. Davidson, and W. Ostertag. 1976. Size, subunit composition, and secondary structure of the Friend virus genome. J. Virol. 20:264-272.

13. Duesberg, P. H. 1968. Physical properties of Rous sarcoma virus RNA. Proc. Natl. Acad. Sci. U.S.A. 68:1511-1518.

14. Erikson, R. L. 1969. Studies on the RNA from avian myeloblastosis virus. Virology 37:454-459.

15. Hartley, J. W., and W. P. Rowe. 1966. Production of altered cell foci in tissue culture by defective Moloney sarcoma virus particles. Proc. Natl. Acad. Sci. U.S.A. 55:780-786.

16. Haseltine, W. A., A. M. Maxam, and W. Gilbert. 1977. Rous sarcoma virus genome is terminally redundant: the 5 ' sequence. Proc. Natl. Acad. Sci. U.S.A. 74:989-993.

17. Hu, S., N. Davidson, and I. Verma. 1977. A heteroduplex study of the sequence relationship between the RNAs of M-MSV and M-MLV. Cell 10:469-477. 
18. Kung, H. J., S. Hu, W. Bender, J. M. Bailey, and N. Davideon. 1976. RD-114, baboon, and woolly monkey viral RNAs compared in size and structure. Cell 7:609-620.

19. Maisel, J., D. Dina, and P. H. Duesberg. 1977. Murine sarcoma viruses: the helper-independence reported for a Moloney variant is unconfirmed; distinct strains differ in size of their RNAs. Virology 76:295-312.

20. Maisel, J., V. Klement, M. M.-C. Lai, W. Ostertag, and P. Duesberg. 1973. Ribonucleic acid components of murine sarcoma and leukemia viruses. Proc. Natl. Acad. Sci. U.S.A. 70:3536-3540.

21. Maisel, J., E. M. Scolnick, and P. Duesberg. 1975. Base sequence differences between the RNA components of Harvey sarcoma virus. J. Virol. 16:749-753.

22. Mangel, W. F., H. Delius, and P. H. Duesberg. 1974. Structure and molecular weight of the 60-70S RNA and the 30-40S RNA of the Rous sarcoma virus. Proc. Natl. Acad. Sci. U.S.A. 71:4541-4545.

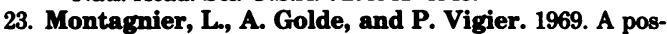
sible subunit structure of Rous sarcoma virus RNA. J. Gen. Virol. 4:449-452.

24. Scolnick, E. M., R. S. Howk, A. Anisowicz, P. T. Peebles, C. D. Scher, and W. P. Parks. 1975. Separation of sarcoma virus-specific and leukemia virus- specific genetic sequences of Moloney sarcoma virus. Proc. Natl. Acad. Sci. U.S.A. 72:4650-4654.

25. Thuring, R., J. P. M. Sanders, and P. Borst. 1975. The freeze-squeeze method for recovering long DNA from agarose gels. Anal. Biochem. 66:213-220.

26. Troxler, D. H., J. K. Boyars, W. P. Parks, and E. M. Scolnick. 1977. Friend strain of spleen focus-forming virus: a recombinant between mouse type $\mathrm{C}$ ecotropic viral sequences and sequences related to xenotropic virus. J. Virol. 22:361-372.

27. Vogt, $P$. K., and P. H. Duesberg. 1973. On the mechanism of recombination between avian RNA tumor viruses. In W. S. Robinson and F. Fox (ed.), Virus research: second ICN-UCLA symposium. Academic Press Inc., New York.

28. Wang, L.-H., and P. Duesberg. 1974. Properties and location of $\operatorname{poly}(\mathrm{A})$ in Rous sarcoma virus RNA. J. Virol. 14:1515-1529.

29. Weiss, R., W. Mason, and P. K. Vogt. 1973. Genetic recombination between endogenous and exogenous avian RNA tumor viruses. Virology 52:535-552.

30. Yuen, P. H., and P. K. Y. Wong. 1977. A morphological study on the ultrastructure and assembly of murine leukemia virus using a temperature-sensitive mutant restricted in assembly. Virology 80:260-274. 